\title{
The effect of exercise on innate mucosal immunity
}

Nicholas P West, David B Pyne, Jennelle M Kyd, Gillian MC Renshaw, Peter A Fricker and Allan W Cripps

Br. J. Sports Med. published online 22 May 2008;

doi:10.1136/bjsm.2008.046532

Updated information and services can be found at:

http://bjsm.bmj.com/cgi/content/abstract/bjsm.2008.046532v1

\section{These include:}

Rapid responses You can respond to this article at:

http://bjsm.bmj.com/cgi/eletter-submit/bjsm.2008.046532v1

Email alerting Receive free email alerts when new articles cite this article - sign up in the box at the service top right corner of the article

\section{Notes}

Online First contains unedited articles in manuscript form that have been peer reviewed and accepted for publication but have not yet appeared in the paper journal (edited, typeset versions may be posted when available prior to final publication). Online First articles are citable and establish publication priority; they are indexed by PubMed from initial publication. Citations to Online First articles must include the digital object identifier (DOIs) and date of initial publication.

To order reprints of this article go to:

http://journals.bmj.com/cgi/reprintform

To subscribe to British Journal of Sports Medicine go to:

http://journals.bmj.com/subscriptions/ 


\section{The effect of exercise on innate mucosal immunity}

N.P. West,${ }^{* \dagger}$ D.B. Pyne, ${ }^{* \ddagger}$ J.M. Kyd, ${ }^{\delta}$ G.M.C. Renshaw, ${ }^{\dagger}$ P.A. Fricker, ${ }^{* *}$ A.W. Cripps ${ }^{*}$

*Department of Physiology, Australian Institute of Sport, 'School of Physiotherapy and Exercise Science, Griffith Health, Griffith University, ${ }^{*}$ School of Medicine, Australian National University, ${ }^{\delta}$ School of Health Services, Central Queensland University, ${ }^{* *}$ Executive, Australian Institute of Sport, ${ }^{\star}$ Griffith Health, Griffith University, Australia.

Keywords: Lactoferrin, lysozyme, exercise, mucosal immunity

Corresponding author:

Professor Allan W Cripps

Griffith Health

Griffith University

Gold Coast campus

Griffith University 4222, Queensland, Australia

The Corresponding Author has the right to grant on behalf of all authors and does grant on behalf of all authors, an exclusive licence (or non exclusive for government employees) on a worldwide basis to the BMJ Publishing Group Ltd and its Licensees to permit this article (if accepted) to be published in Journal (British Journal of Sports Medicine) editions and any other BMJPGL products to exploit all subsidiary rights, as set out in our licence (http://bjsm.bmjjournals.com/misc/ifora/licenceform.shtml).

I declare that neither I nor the co-authors have any competing interests 


\begin{abstract}
We conducted a prospective observational study comparing salivary lactoferrin and lysozyme concentration over five months (chronic changes) in elite rowers $(n=17$, mean age $24.3 \pm 4.0 \mathrm{y})$ with sedentary individuals (controls) $(n=18$, mean age $=27.2 \pm 7.1 \mathrm{y})$ and a graded exercise test to exhaustion (acute changes) with a cohort of elite rowers $(n=11$, mean age $24.7 \pm 4.1)$. Magnitudes of differences and changes were interpreted as a standardized (Cohen's) effect size (ES). Lactoferrin concentration in the observational study was approximately $60 \%$ lower in rowers than control subjects at baseline $(7.9 \pm 1.2$ $\mu \mathrm{g} . \mathrm{ml}^{-1}$ mean $\pm \mathrm{SEM}, 19.4 \pm 5.6 \mu \mathrm{g} \cdot \mathrm{ml}^{-1}, P=0.05, \mathrm{ES}=0.68$, 'moderate') and at the midpoint of the season $\left(6.4 \pm 1.4 \mu \mathrm{g} \cdot \mathrm{ml}^{-1}\right.$ mean \pm SEM, $21.5 \pm 4.2 \mu \mathrm{g} \cdot \mathrm{ml}^{-1}, P=0.001$, $\mathrm{ES}=0.89$, 'moderate'). The concentration of lactoferrin at the end of the study was not statistically significant $(P=0.1)$ between the groups. There was no significant difference between rowers and control subjects in lysozyme concentration during the study. There was a $50 \%$ increase in the concentration of lactoferrin $(P=0.05, \mathrm{ES}=1.04$, 'moderate') and $55 \%$ increase in lysozyme ( $P=0.01, \mathrm{ES}=3.0$, 'very large') from pre-exercise to exhaustion in the graded exercise session. Lower concentrations of these proteins may be indicative of an impairment of innate protection of the upper respiratory tract. Increased salivary lactoferrin and lysozyme concentration following exhaustive exercise may be due to a transient activation response that increases protection in the immediate post exercise period.
\end{abstract}




\section{INTRODUCTION}

Athletes undertaking prolonged intense exercise, particularly elite athletes, may suffer a higher incidence of URTI than individuals participating in moderate exercise or no exercise at all ${ }^{1,2}$. While the results have been inconclusive, athletes who remain free of illness immediately prior to and during competition are likely to perform better than their peers with illness ${ }^{3}$. Immune resilience is an important factor in success at the elite level ${ }^{4}$ and underpins efforts to understand the mechanisms that regulate susceptibility to illness from exercise.

There have been few investigations into the effect of exercise on innate mucosal immune secretions, particularly antimicrobial proteins (AMPs). AMPs are small cationic proteins that are constituent and inducible factors of mucosal secretions. Two of the most abundant AMPs in mucosal secretions of the upper respiratory tract (URT) are lactoferrin and lysozyme and both act directly against pathogenic organisms ${ }^{5}$. There is increasing interest in the role these proteins play in protecting the body from illness ${ }^{5,6}$.

Measurement of AMPs have been proposed for use as a diagnostic marker of mucosal immune status $^{6,7}$. A preliminary study by our group indicates that prolonged intense training can elicit a reduction in the concentration of salivary lactoferrin at the onset of a training season, which may be an adaptive response to exercise ${ }^{8}$. A short duration, incremental exercise test to exhaustion and a short duration exercise session at $75 \%$ $\mathrm{VO}_{2 \max }$ resulted in a transient increase in the concentration of salivary lysozyme without affecting salivary flow rate ${ }^{7}$. This contrasts with a significant decrease in salivary lysozyme reported following an intense training session in elite swimmers ${ }^{9}$.

The aim of this study was to further our understanding of the relationship between exercise and innate immunity by describing the timecourse and magnitude of changes in the concentration of salivary lactoferrin and lysozyme in elite rowers over a five-month training season. A second aim was to quantify the time course of acute changes in salivary lactoferrin and lysozyme in response to sub-maximal and maximal exercise. 


\section{MATERIAL AND METHODS \\ Study design}

The prospective observational study ran for five months as a cohort of elite rowers prepared for selection to the Australian Rowing Team. Differences in lactoferrin and lysozyme were assessed between the rowers and a sedentary control group at baseline, the midpoint of the training period and at the completion of the training period. A subgroup of rowers completed a progressive rowing ergometer text to exhaustion to assess the impact of submaximal and maximal exercise loads on salivary AMP concentration under controlled conditions in the laboratory. The control group did not undergo the incremental test to exhaustion as the study did not seek to directly compare whether there was a difference in the response of antimicrobial proteins between elite athletes and sedentary individuals to submaximal and maximal exercise.

\section{Subjects}

There were 17 elite rowers (eight male, ages $25.9 \pm 4.0 \mathrm{y}, \mathrm{VO}_{2 \max } 5.4 \pm 0.4 \mathrm{ml} \cdot \mathrm{kg}^{-1} \cdot \mathrm{min}^{-1}$, nine female, ages $23.8 \pm \mathrm{VO}_{2 \max } 4.2 \pm 0.3 \mathrm{ml} \cdot \mathrm{kg}^{-1} \cdot \mathrm{min}^{-1}$ ) that participated in the longitudinal study. A sedentary control group consisting of eight males and nine females (age $=27.3 \pm 7.2 \mathrm{y}$ ) was also recruited and were restricted to five hours physical activity per week based on physical activity guidelines from the National Physical Activity Guidelines (NPAG) and surveys reporting physical activity levels of the Australian Community $^{10,11}$. A sub-group of 11 elite rowers (four male, age $=27.0 \pm 5.4 \mathrm{y}, \mathrm{VO}_{2 \max }$ $5.6 \pm 0.7 \mathrm{ml} . \mathrm{kg}^{-1} \cdot \mathrm{min}^{-1}$, seven female, age $23.5 \pm 2.8 \mathrm{y}, \mathrm{VO}_{2 \max } 4.9 \pm 0.3 \mathrm{ml} . \mathrm{kg}^{-1} \cdot \mathrm{min}^{-1}$ ) participated in the rowing ergometer test. All subjects were screened for use of immunomodulating medications before inclusion in the project. The study was approved by the Griffith University Human Research Ethics Committee and the AIS Ethics Committee and all participants provided written informed consent.

\section{Saliva collection}

In the observational study samples were taken every two weeks from the rowers prior to an afternoon training session and from the control subjects at baseline, at the mid-point, and the end of the study. All saliva samples in the observational study were taken at the same time of the day to control for diurnal variation and at least one hour post prandial. Saliva samples in the rowing ergometer test were taken pre test, following a moderate exercise load and after a maximal step to exhaustion. The moderate exercise load was determined as the point that blood lactate reached $4 \mathrm{mM}$. Saliva samples were collected using an eye spear (Defries Industies Pty Ltd, Victoria, Australia), which was placed between the cheek and teeth for up to five minutes. All samples were frozen at minus $80^{\circ} \mathrm{C}$ until analysis. Albumin concentration was assessed to control for changes in salivary flow rate.

\section{Training program}

The training program undertaken was a periodised program designed by AIS coaches. The training program had three distinct phases, an initial endurance phase characterized by high volume and low intensity, a mid-season quality phase of moderate volume and high intensity training, and a taper phase leading into the selection events. An outline of the training program corresponding to weeks 4 to 11 in the longitudinal study is shown in Figure 1. No training details were available for the initial three weeks of the study, from late December to early January. The athletes maintained a daily illness log that recorded 
the details for the sessions undertaken to allow comparison to the designed training program.

\section{Submaximal and Maximal Effort Rowing Test}

This rowing ergometer test involves the completion of several steps, each of four minutes duration at increasing intensity and with each step separated by a one minute recovery interval. The graded exercise test has been described in detail previously ${ }^{12}$. Each subject completed the number of steps required to reach $4 \mathrm{mM}$ lactate in blood or until seven steps was attained. $4 \mathrm{mM}$ is a commonly utilised approximation of the mean lactate steady state and was therefore nominated as the submaximal workload. Once blood lactate reached $4 \mathrm{mM}$ or the rower has completed seven steps the athlete had a 20 minute rest period before completing an Olympic distance step at maximal exertion. A series of calculations based on the subject's best previous 2000 metre rowing ergometer time was used to individualise the workload for the steps undertaken until blood lactate reaches four mM.

\section{Lactoferrin, lysozyme and albumin assays}

The concentration of salivary lactoferrin and lysozyme was measured in duplicate using a commercial ELISA assay kits (lactoferrin - Calbiochem, Darmstadt, Germany and lysozyme - ImmuneDiagnostik, Bensheim, Germany) according to the manufacturer's instructions. All saliva samples were centrifuged before analysis. The following dilutions were made: lactoferrin 1:600 rowers and 1:800 controls; lysozyme 1:1500 rowers and 1:3000 controls to produce values within the standard curve. Dilution factors were optimized for each group to ensure an acceptable goodness of fit to the standard curve. The concentration of both proteins was measured colorimetrically at 450nm on a BioRad Model 3550 Microplate Reader (Bio-Rad, California, USA). All samples were analysed in a single batch to reduce the variability of the measurements. The between-run coefficient of variation (CV) was $8.8 \%$ for lactoferrin and $9.9 \%$ for lysozyme which are similar to levels reported previously ${ }^{13}$. Albumin concentration was measured using a commercial albumin kit (Roche/Hitachi, Mannheim, Germany) on a Hitachi 911 analyser (Hitachi, Boehringer, Mannheim, Germany). Each sample was diluted 1:10. The sensitivity range on the test is from $3-400 \mathrm{mg} / \mathrm{L}$.

\section{Statistical analysis}

Data was log-transformed as is usual practice for immunological measures in saliva. Data is presented as mean \pm standard error of the mean. The primary analysis involved a comparison of the change in mean differences between the athlete and control groups using an independent t-test with unequal variance. The repeated measures analysis of fortnightly changes in lactoferrin and lysozyme in the rowers was undertaken by comparing the change in the means between the experimental and control groups ${ }^{14}$. For the rowing ergometer test a pair-wise comparison of the pre-, mid-, and post-exercise sampling points was conducted using an independent t-test. We used the approach of controlled trial analysis outlined by Batterham ${ }^{15}$. The magnitudes of changes in AMP concentration were expressed as a standardized mean (Cohens) difference (difference in means divided by the between-subject standard deviation). The criteria to interpret the magnitude of the effect sizes were: $<0.2$ trivial, 0.2-0.6 small, 0.6-1.2 moderate, 1.2-2.0 large and $>2.0$ very large. Significance was accepted at the level $P<0.05$. To compare the magnitude of variation in lactoferrin and lysozyme concentrations between the rowers 
and controls we divided the between-subject coefficient of variations $(\mathrm{CV})$. Ratios within a range of 0.9-1.1 were considered trivial (see justification at

http://yahoogroups.com/groups/sportscience/message/2538). Ratios >1.1 indicate AMP concentrations were substantially more variable for the rowers, whereas ratios $<0.9$ indicates AMP concentrations were substantially less variable for the rowers than the control subjects.

\section{RESULTS}

\section{Mean concentration of lactoferrin in saliva}

The mean concentration and standard error of the mean for salivary lactoferrin in the control subjects and the rowers is shown in Figure 2. The concentration of lactoferrin in the rowers was $\sim 60 \%$ lower than control subjects at the start of the season $(P=0.05$, $\mathrm{ES}=0.68$, 'moderate' $)$ and the midpoint $(P=0.01, \mathrm{ES}=0.89$, 'moderate' $)$. While the difference was not significant at the end point of the study $(P=0.12, \mathrm{ES}=0.53$, 'small') the concentration of salivary lactoferrin was half that of the control subjects. There were no significant temporal changes in the concentration of salivary lactoferrin in the rowers during the training period. While the concentration of salivary lactoferrin in both groups was characterized by large variation the rowers had a similar within-group variation $(58 \%)$ compared to the control subjects $(52 \%)$ : ratio of $C V=1.12$. There was substantially greater between-group variation for the controls $(107 \%)$ than the rowers $(\mathrm{CV}=77 \%)$ ratio of $\mathrm{CV}=0.73$. There was no substantial difference in the concentration of albumin between the two groups. Correcting for albumin had little effect on the outcomes of the study.

\section{Mean concentration of lysozyme in saliva}

The mean concentration and standard error of the mean for salivary lysozyme for the rowers and control subjects is shown in Figure 3. There was a non-significant difference in the concentration of lysozyme throughout the study between rowers and controls. There was similar within-subject variability in the concentration of lysozyme in control subjects $(67 \%)$ and rowers $(71 \%$, ratio of $\mathrm{CV}=1.06)$. The between-group variations were also similar (controls $120 \%$, rowers $109 \%$, ratio of CV 0.90). Correcting for albumin had no effect on the results.

Acute response of salivary lactoferrin and lysozyme to rowing ergometry There was a 50\% increase in mean salivary lactoferrin concentration from pre-test (15.3 $\pm 3.0 \mu \mathrm{g} . \mathrm{ml}^{-1}$ mean $\left.\pm \mathrm{SEM}\right)$ to the maximal exertion step $\left(30.2 \pm 5.1 \mu \mathrm{g} . \mathrm{ml}^{-1}\right)$ that was statistically significant ( $P=0.04, \mathrm{ES}=1.04$, 'moderate'). There was no increase in the concentration of lactoferrin from pre-test to the submaximal step $\left(15.6 \pm 5.1 \mu \mathrm{g} \cdot \mathrm{ml}^{-1}\right.$, $P=0.95)$ There was a significant linear increase $(122 \%)$ in mean salivary lysozyme concentration from pre-test $\left(13.5 \pm 3.8 \mu \mathrm{g} . \mathrm{ml}^{-1}\right)$ to post test $\left(29.6 \pm 3.9 \mu \mathrm{g} . \mathrm{ml}^{-1}, P=0.01\right.$, $\mathrm{ES}=3.0$, 'large'). There was a non-significant $(P=0.14) 60 \%$ increase in the concentration of lysozyme from pre-test to mid test $\left(21.7 \pm 3.8 \mu \mathrm{g} . \mathrm{ml}^{-1}\right)$.

\section{DISCUSSION}

This study found that elite rowers had approximately half the concentration of salivary lactoferrin and lysozyme than non-exercising control subjects over a five month training season. There was, however, no substantial temporal change in the concentration of these proteins in the rowers over the observation period. The concentrations of the proteins in 
both studies were marked by large within subject (range 100-300\%) and between group (range 50-150\%) variations. Humoral factors of the innate immune system are central to host resistance ${ }^{16}$. Lactoferrin and lysozyme play a complex role in host defence and there is little understanding of the way in which changes in their concentration affect susceptibility to upper respiratory tract infection in-vivo ${ }^{5,17}$. These data indicate that prolonged intense rowing training lowers the concentration of a key salivary protein of innate mucosal immunity, which might leave individuals at greater risk of contracting illness and negatively impact training and competitive performance.

In this study episodes and severity of illness were recorded. However, there were too few illnesses reported over the observation period for any meaningful statistical analysis to be conducted. A larger study, powered to determine differences in infections between the two groups, is required.

Assessing a range of analytes, including differential cell counts, cell functional activity and both innate and adaptive humoral factors, may provide a more accurate picture of the way changes in immunity are associated with susceptibility to illness. Examining the variability of mucosal secretions of athletes in response to training and exercise may also hold promise in gaining a further understanding of the relationship between exercise, immunity and illness. The large variations in the concentrations of both the AMP proteins are consistent with studies of other mucosal immune parameters. Traditionally such large variability has been viewed as a limiting factor in determining the likelihood of detecting real change in the parameters being examined ${ }^{18}$. Our findings indicate that regular physical exercise (athletic training) reduces the variability of both lactoferrin and lysozyme. A substantial difference in an individual's variability from the group's variability might be indicative of mucosal immune modulation and a subsequent change in susceptibility to illness ${ }^{19}$. Expanding the number of immune parameters examined may yield a simple test that can be used by athletes and coaches to provide further information on the immunological status of the athlete.

Our inability to identify substantial temporal change in the concentration of salivary lactoferrin contrast with an earlier study that showed an initial sharp reduction at the onset of an exercise training period, which then remained low for the duration of the training period ${ }^{8}$. These contrasting findings may be due to the fact that in the current study the athletes had been training prior to the first sample being collected while in the earlier study the athletes began training after being enrolled. As such, the initial decline in salivary lactoferrin and lysozyme concentration may have already occurred. These disparate findings highlight the importance of understanding a subject's recent training history when undertaking such studies. The stability in AMP concentration contrasts with the acute and chronic decreases described for salivary immunoglobulin A with prolonged intense exercise $(\mathrm{SIgA})^{20}$. This difference may relate to the underlying immune regulation of antimicrobial peptides and the adaptation of the athletes to the training load. The persistent low basal concentrations of the proteins in this study may be a function of altered autonomic nervous system activity induced by twice daily exercise. There is strong involvement of the neuroendocrine system in the regulation of innate secretory immunity ${ }^{21}$. Prolonged intense exercise is associated with activation of the hypothalamicpituitary-adrenal axis and the secretion of glucocorticoids, with the exercise induced changes in the concentration of these neuropeptides persisting for several hours post 
exercise. Increased secretion of glucocorticoids is associated with a reduction in salivary lysozyme concentration ${ }^{7}$. It is plausible that elevated cortisol levels in the post exercise period may be the underlying mechanism leading to reduced concentration of lactoferrin and lysozyme in saliva. Examining changes in salivary lactoferrin and lysozyme with other indices of stress and adaptation may yield further information about whether changes over a training period are related to the body's ability to tolerate the exercise load.

In contrast to the findings from the longitudinal study, there was a significant increase in the acute response of lactoferrin and lysozyme in response to a graded exercise test to exhaustion. The findings of an increase in salivary lactoferrin and lysozyme following maximal exertion exercise are consistent with a recent study that reported a $160 \%$ increase in salivary lysozyme concentration in response to both an acute exercise session at $75 \% \mathrm{VO}_{2 \max }$ and at maximal exertion ${ }^{7}$. Interestingly, we found that there was a nonsignificant increase in the concentration of salivary lysozyme but not lactoferrin in response to a submaximal workload. This suggests that there may be an additive effect as the intensity of exercise increases on the concentration of salivary AMPs. It has been postulated that exercise-induced increases in the concentration of salivary AMPs are most likely related to sympathetic nervous system activity ${ }^{7}$. The increase in the concentration of these salivary proteins, particularly in response to maximal exertion exercise, may also have been the result of damage to epithelial cells through hyperventilation and subsequent exposure to environmental irritants, and the activation of neutrophils that then follows. Epithelial cells increase their expression of AMPs following physical damage ${ }^{22}$ and contact with microbes ${ }^{23}$. It has also been postulated that increased concentration of AMPs following exhaustive exercise might confer improved immunity to infection. This view conflicts with the prevailing view, however, that immune competence is reduced and susceptibility to opportunistic infection increased immediately following prolonged exhaustive exercise ${ }^{24}$. The findings of this acute study indicate that it is high intensity exercise of long duration that is more likely to lead to immune suppression in the post exercise period.

In summary, this study provides evidence that elite rowers have chronically reduced concentrations of lactoferrin and lysozyme in the upper respiratory tract in comparison to their non-exercising counterparts. This study also found a transient activation response whereby AMPs increase in concentration in response to acute submaximal and maximal exertion exercise. The significance of the transient increase is yet to be determined. Future studies need to examine a range of exercise-induced changes to mucosal immunity and their effect on susceptibility to infection in a multi-factorial model. The use of a multifactorial model may provide insight into the way in which exercise-induced mucosal perturbation is related to susceptibility to illness.

\section{ACKNOWLEDGEMENTS}

The authors thank Sharon Hall, Vicki Brent and Amanda Cox, the athletes and coaches of the AIS and ACTAS and Sue Gibbons and Karen Noller for their assistance during the project. 


\section{What is already known on this topic?}

Salivary antimicrobial proteins (AMPs) have been proposed as markers of stress on the innate immune system. Contrasting findings have been reported on the effects of acute exercise on AMPs. No published work has examined the chronic effects of exercise on base concentrations of AMPs.

\section{What this study adds?}

Elite rowers have a $\sim 50 \%$ lower concentration of salivary AMPs compared to sedentary individuals. There is large intra and inter-individual variability in the concentration of these proteins in both rowers and sedentary groups. More work is required to determine the clinical significance of lower salivary AMP concentration in athletes.

\section{FIGURE LEGENDS}

Figure 1: Schematic diagram showing the weekly training load undertaken by the rowers during the study.

Figure 2: Salivary lactoferrin levels in rowers $(\boldsymbol{\square}-\boldsymbol{\square})$ and control $(\boldsymbol{\Delta}-\boldsymbol{\Delta})$ subjects. Values presented are the mean \pm standard error of the mean. $P$ values indicate statistical significance at the commencement (baseline), midpoint and end of the study.

Figure 3: Salivary lysozyme levels in rowers ( $\boldsymbol{\square}-\boldsymbol{\square})$ and control $(\boldsymbol{\Delta}-\boldsymbol{\Delta})$ subjects. Values presented are the mean \pm standard error of the mean. 


\section{REFERENCES}

1. Reid, V. L.; Gleeson, M.; Williams, N.; Clancy, R. L., Clinical investigation of athletes with persistent fatigue and/or recurrent infections. Br J Sports Med 2004, $38,(1), 42-5$.

2. Konig, D.; Grathwohl, D.; Weinstock, C.; Northoff, H.; Berg, A., Upper respiratory tract infection in athletes: influence of lifestyle, type of sport, training effort, and immunostimulant intake. Exerc Immunol Rev 2000, 6, 102-20.

3. Pyne, D.; McDonald, W.; Gleeson, M.; Flanagan, A.; Clancy, R.; Fricker, P., Mucosal immunity, respiratory illness, and competitive performance in elite swimmers. Med Sci. Sports Exerc 2000, 33, (3), 348-353.

4. Malm, C., Exercise immunology: the current state of man and mouse. Sports Med 2004, 34, (9), 555-66.

5. Laube, D. M.; Yim, S.; Ryan, L. K.; Kisich, K. O.; Diamond, G., Antimicrobial peptides in the airway. Curr Top Microbiol Immunol 2006, 306, 153-82.

6. West, N. P.; Pyne, D. B.; Renshaw, G.; Cripps, A. W., Antimicrobial peptides and proteins, exercise and innate mucosal immunity. FEMS Immunol Med Microbiol 2006, 48, (3), 293-304.

7. Allgrove, J. E.; Gomes, E.; Hough, J.; Gleeson, M., Effects of exercise intensity on salivary antimicrobial proteins and markers of stress in active men. Journal of Sports Sciences 2008, 26, (6), 653-661.

8. Cox, G.; Pyne, D.; Kyd, J.; McDonald, W.; Fricker, P.; Cripps, A., Salivary lactoferrin as a marker of immunocompetence in elite swimmers. Int J Sports Med 1999, 21, (S1), S83-84.

9. Inoue, H.; Sakai, M.; Kaida, Y.; Kaibara, K., Blood lactoferrin release induced by running exercise in normal volunteers: antibacterial activity. Clin Chim Acta 2004, 341, (1-2), 165-72.

10. Vasianovich, A.; van Teijlingen, E. R.; Reid, G.; Scott, N. W., Key health promotion factors among male members of staff at a higher educational institution: a cross-sectional postal survey. BMC Public Health 2008, 8, (1), 58.

11. Welfare, A. I. o. H. a., Indicators for chronic diseases and their determinants. Cat. no. PHE 75. Canberra: AIHW. 2008.

12. Vogler, A.; Rice, A.; R, W., Physiological responses to exercise on different models of Concept II rowing ergometer. Int J Sports Physiol Perform 2007.

13. Taylor, D. C.; Cripps, A. W.; Clancy, R. L., Measurement of lysozyme by an enzyme-linked immunosorbent assay. Journal of Immunological Methods 1992, $146,55-61$.

14. Hopkins, W. G. A new view of statistics. Internet Society for Sport Science. http://www.sportsci.org/resource/stats/ (16/5/2007),

15. Batterham, A.; Hopkins, W., A decision tree for controlled trials. Sports Science 2005, 9, 33-39. 
16. Amerongen, A. V.; Veerman, E. C., Saliva--the defender of the oral cavity. Oral Dis 2002, 8, (1), 12-22.

17. Roxas, M.; Jurenka, J., Colds and influenza: a review of diagnosis and conventional, botanical, and nutritional considerations. Altern Med Rev 2007, 12 , (1), 25-48.

18. Hopkins, W. G., Measures of reliability in sports medicine and science. Sports Med 2000, 30, (1), 1-15.

19. Francis, J.; Gleeson, M.; Pyne, D.; Callister, R.; Clancy, R., Variation of salivary immunoglobulins in exercising and sedentary populations. Med Sci Sports Exerc 2005, 37, (4), 571-8.

20. Gleeson, M.; McDonald, W. A.; Pyne, D. B.; Cripps, A. W.; Francis, J. L.; Fricker, P. A.; Clancy, R. L., Salivary IgA levels and infection risk in elite swimmers. Med Sci Sports Exerc 1999, 31, (1), 67-73.

21. Bosch, J. A.; de Geus, E. J.; Veerman, E. C.; Hoogstraten, J.; Nieuw Amerongen, A. V., Innate secretory immunity in response to laboratory stressors that evoke distinct patterns of cardiac autonomic activity. Psychosom Med 2003, 65, (2), 245-58.

22. Dorschner, R. A.; Pestonjamasp, V. K.; Tamakuwala, S.; Ohtake, T.; Rudisill, J.; Nizet, V.; Agerberth, B.; Gudmundsson, G. H.; Gallo, R. L., Cutaneous injury induces the release of cathelicidin anti-microbial peptides active against group $\mathrm{A}$ Streptococcus. J Invest Dermatol 2001, 117, (1), 91-7.

23. Duits, L. A.; Nibbering, P. H.; van Strijen, E.; Vos, J. B.; Mannesse-Lazeroms, S. P.; van Sterkenburg, M. A.; Hiemstra, P. S., Rhinovirus increases human betadefensin-2 and -3 mRNA expression in cultured bronchial epithelial cells. FEMS Immunol Med Microbiol 2003, 38, (1), 59-64.

24. Nieman, D., Is infection risk linked to exercise workload? Med Sci Sports Exerc 2000, 32, (7), S406-11. 
Figure 1

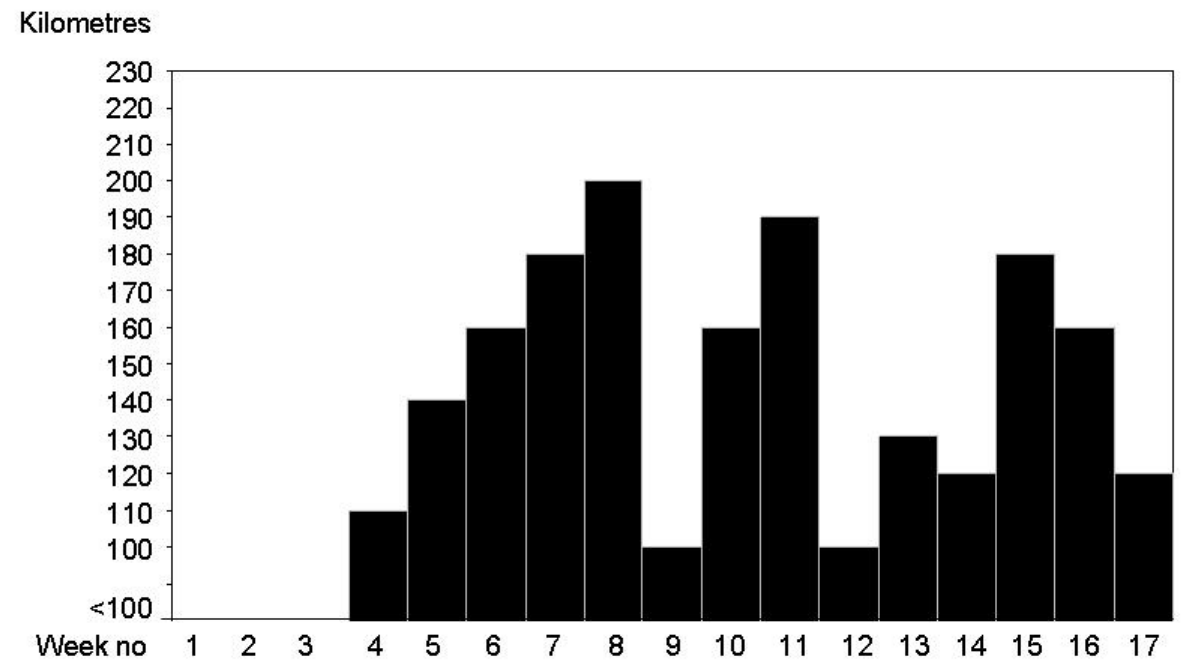


Figure 2

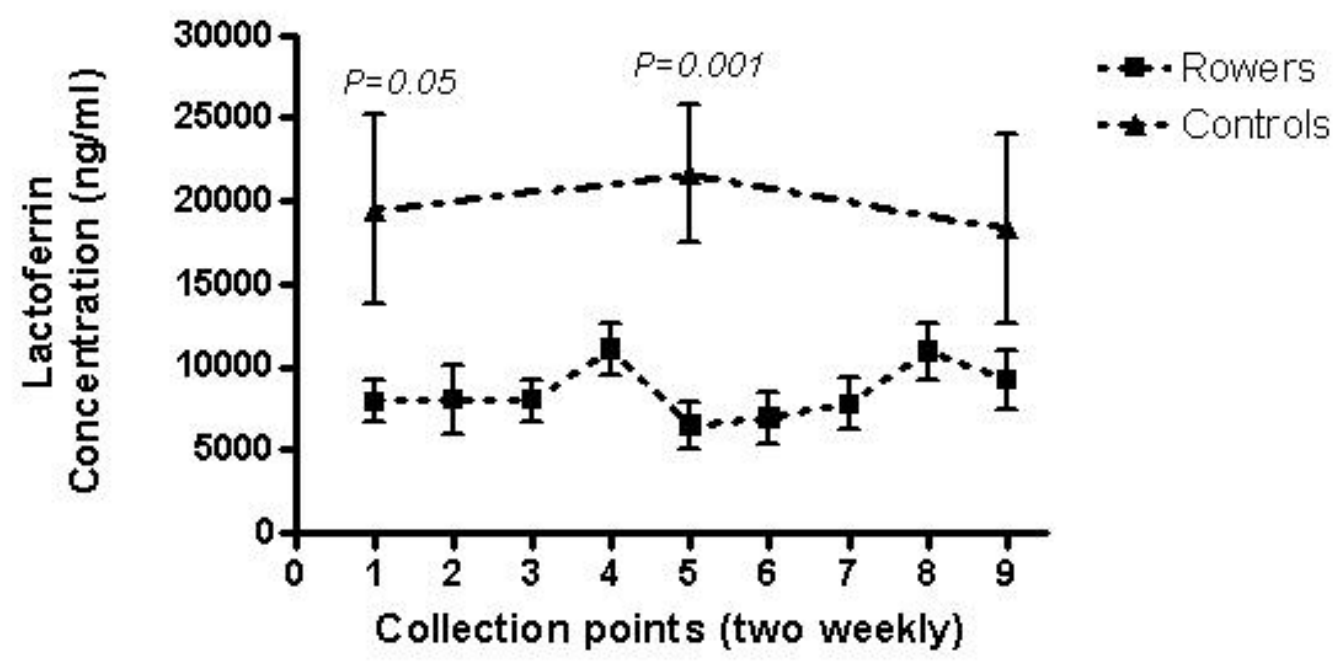


Figure 3

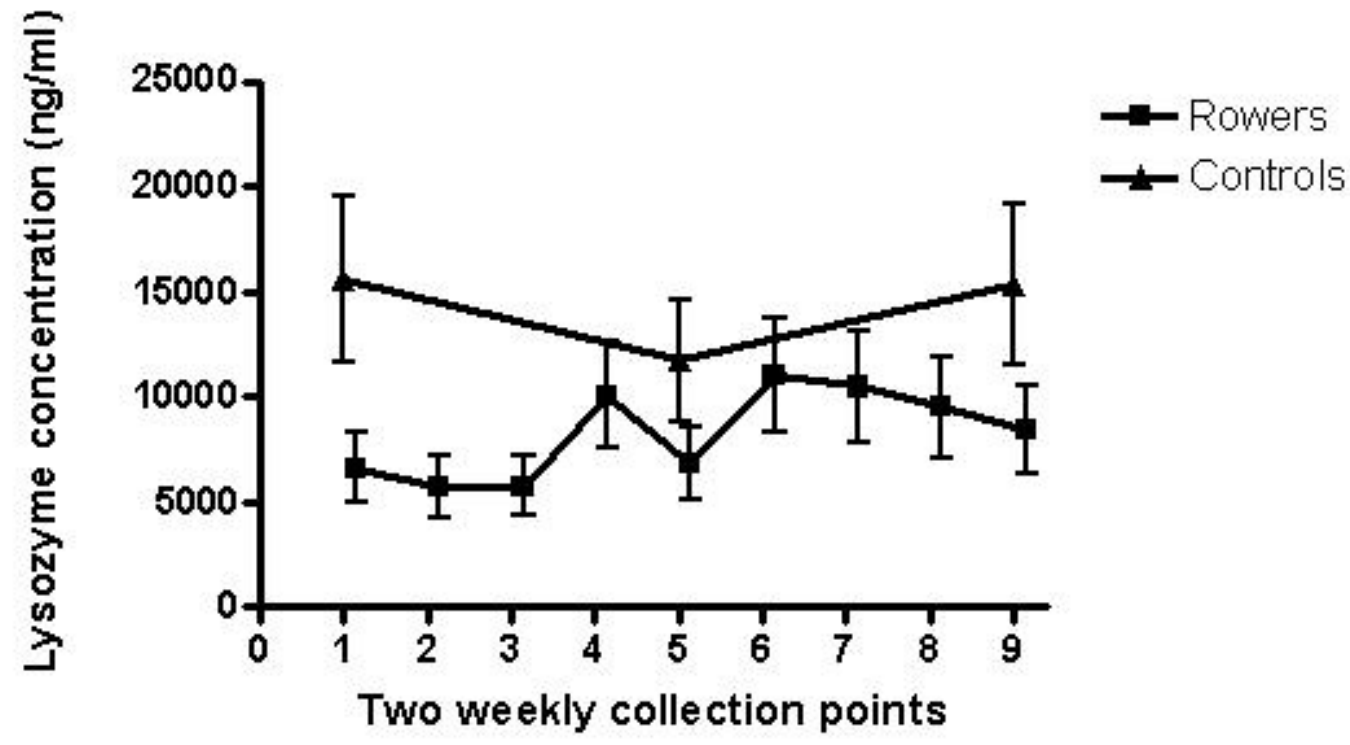

